Received 00th January 20xx, Accepted 00th January 20xx

DOI: $10.1039 / \mathrm{x} 0 \mathrm{x} \times 00000 \mathrm{x}$

www.rsc.org/

\title{
New Pyrene Cored Small Organic Molecule with Flexible Alkyl Spacer: The Potential Solution Processable Blue Emitter with Bright Photoluminescence
}

\author{
Nitin Valsange, ${ }^{a}$ F. L. Wong, ${ }^{b}$ Durgaprasad Shinde, ${ }^{a}$ C. S. Lee, ${ }^{b}$ V. A. L. Roy, ${ }^{b}$ Sergei Manzhos, ${ }^{c}$ \\ K. Feron, ${ }^{\mathrm{d}}$ Samuel Chang, ${ }^{\mathrm{e}}$ Ryuzi Katoh, ${ }^{\mathrm{e}}$ Prashant Sonar ${ }^{\mathrm{f} *}$ and Prakash P. Wadgaonkar ${ }^{\mathrm{a} *}$
}

\begin{abstract}
A new pyrene cored small organic molecule viz., 1,3,6,8-tetrakis(4-((5-(9H-carbazol-9yl)pentyl)oxy)phenyl)pyrene (PY-II) was designed and synthesized. The carbazole moiety with alkyl spacer was introduced at 1, 3, 6 and 8 positions of pyrene core to improve the charge transport properties and solution processability. PY-II exhibited excellent solubility in common organic solvents and high thermal stability up to $345^{\circ} \mathrm{C}$. The photoluminescence quantum yield (PLQY) of PY-II in solution was found to be 0.9 with bright blue emission near $450 \mathrm{~nm}$ which is just appropriate to human eye. The solution processed non-doped OLED device fabricated using PY-II as an emissive layer afforded a pure blue emission with CIE coordinates of 0.16 and 0.16 , the power efficiency of $0.17 \mathrm{~lm} \mathrm{~W} \mathrm{~W}^{-1}$, maximum current efficiency of $0.41 \mathrm{~cd} / \mathrm{A}$ and maximum brightness of $202 \mathrm{cdm}^{-2}$.
\end{abstract}

\section{Introduction}

Over the past two decades, organic light emitting diodes (OLEDs) have attracted great attention because of their applications in flat panel displays and solid-state lighting devices. ${ }^{1-5}$ Generally, small molecule organic light emitting diodes (SMOLEDs) are fabricated by high-vacuum thermal evaporation whereas polymer light emitting diodes (PLEDs) are fabricated by the solution processing method. Vacuum evaporation results in complicated architectures, is expensive and is not well suited for large area fabrication. ${ }^{6,7}$ On the other hand, solution processing is an attractive method with low cost and large area manufacturability. ${ }^{8-11}$ To date, both small molecules and polymers have been extensively used as emitting materials in OLEDs. ${ }^{11-14}$ Recently, OLEDs based on organic and organometallic compounds have drawn intense interest due to their inherent features such as excellent solution processability, tunable emission properties, thin film forming ability and high chemical and thermal stability. A variety of solution processable fluorescent and phosphorescent light emitting materials have been reported to date. ${ }^{14-18}$

Among the various fluorescent chromophores used for OLEDs, pyrene stands out as a promising chromophore because

\footnotetext{
a. Polymer Science and Engineering Division, CSIR-National Chemical Laboratory, Dr. Homi Bhabha Road, Pune 411008, India.

b. Center of Super Diamond and Advanced Films, and Department of Biology and Chemistry, City University of Hong Kong, Tat Chee Avenue, Kowloon, Hong Kong

c. Department of Mechanical Engineering, Faculty of Engineering, National

University of Singapore Block EA \#07-08, 9 Engineering Drive 1, Singapore 117576

d. CSIRO Energy Centre, 10 Murray Dwyer Circuit, Mayfield West, NSW 2304,

Australia

e. Department of Chemical Biology and Applied Chemistry, College of Engineering,

Nihon University, Koriyama, Fukushima 963-8642, Japan

f. School of Chemistry Physics and Mechanical Engineering, Queensland University

of Technology (QUT) 4001 Brisbane, Australia.
}

Electronic Supplementary Information (ESI) available: [details of any supplementary information available should be included here]. See DOI: $10.1039 / \times 0 \times x 00000 x$ of its strong emission efficiency and high charge carrier mobility. ${ }^{19-22}$ These features make pyrene a potential candidate to develop an interesting class of materials for OLEDs. However, supramolecular $\pi-\pi$ stacking between pyrene cores in the solid state leads to the red shift and fluorescence quenching of the emission. ${ }^{23,24}$ To overcome these problems, rigid and bulky groups with alkyl chains were introduced at the periphery of pyrene core which prevent the intermolecular aggregation and lead to enhanced emission efficiency. ${ }^{25}$ A number of light emitting materials based on pyrene have been reported in the literature. ${ }^{26-29}$ Recently, we reported carbazole and phenothiazine containing solution processable blue and green emitting 1, 3, 6, 8-tetrasubstituted pyrene derivatives. ${ }^{27}$ Pyrene has been successfully utilized for synthesis of materials with pure blue and green emission. ${ }^{30-32}$ Blue light-emitting materials can facilitate white light emission in the presence of suitable dopants. ${ }^{33-36}$ Thus, it is important to develop new classes of blue emitting materials with good solution processability and high fluorescence efficiency. It is reported that moieties with charge transporting ability and aliphatic chain linkers offer several advantages such as enhancement in the solubility, facilitated charge transport and reduction of intermolecular interaction between the emissive cores. ${ }^{37-41}$ Such molecules provide a pathway to reduce or eliminate aggregation induced fluorescence quenching by the site isolation effect. 
(a)

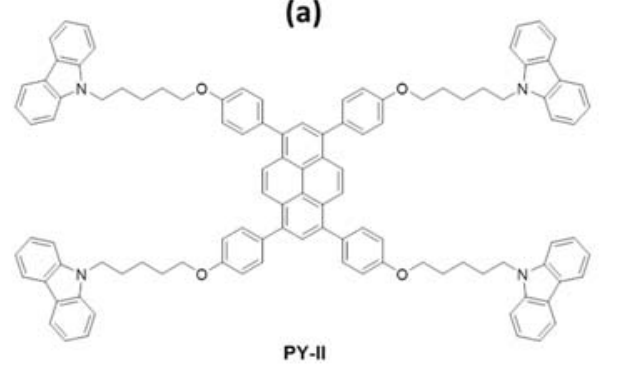

(b)

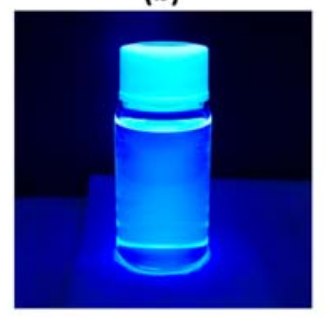

(c)

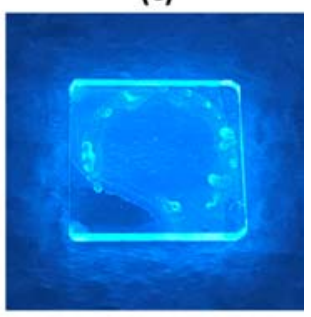

Fig. 1 Chemical structure of PY-II (a), emission under a UV-vis lamp using PY-II in solution (b) \& spin coated thin film of PY-II deposited on quartz plate @ 1500 RPM, 30s using 0.5 mM chloroform solution (c).

According to the above considerations, herein, we report a new pyrene cored solution processable blue light emitting material for OLED devices. The carbazole moiety with alkyl spacer was introduced at 1, 3, 6, 8-positions of the pyrene core. Carbazole was employed due to its high hole transporting and energy harvesting ability, ${ }^{42-46}$ and its property to enhance the emission of the core. ${ }^{37,41,47}$ The flexible alkyl chain was introduced to further improve the solution processability and glass-like properties. ${ }^{7}$ The OLED device fabricated using PY-II (Fig. 1) as an emitting material showed blue emission with a maximum brightness of $202 \mathrm{cdm}^{-2}$ and a maximum current efficiency of $0.41 \mathrm{~cd} / \mathrm{A}$.

\section{Experimental}

\section{Chemicals and Characterizations}

All the chemicals and solvents were purchased from SigmaAldrich and were used without further purification. All the reactions were carried out using a round bottom flask or a Schlenk tube in an argon or nitrogen atmosphere in anhydrous solvents. ${ }^{1} \mathrm{H}$ and ${ }^{13} \mathrm{C}$ NMR spectra were recorded in $\mathrm{CDCl}_{3}$ on a Bruker AC spectrometer operating at $400 \mathrm{MHz}$ for ${ }^{1} \mathrm{H}$ and 100 $\mathrm{MHz}$ for ${ }^{13} \mathrm{C}$. Matrix assisted laser desorption/ionization timeof flight (MALDI-TOF) mass spectra were obtained on a Bruker Autoflex TOF/TOF instrument using dithranol as a matrix. UV-vis absorbance and photoluminescence (PL) spectra were recorded on a Jasco V-570, and a Cary Eclipse Fluorescence spectrometer, respectively, using chloroform as a solvent. Thermogravimetric and DSC analyses were performed on PerkinElmer STA 6000 and TA Instrument DSC Q10, respectively at a heating rate of $10{ }^{\circ} \mathrm{C} \mathrm{min}{ }^{-1}$ under nitrogen atmosphere. The cyclic voltammogram was recorded on Autolab using conventional three-electrode assembly with a Pt wire as a working electrode, a Pt foil as a counter electrode and $\mathrm{Ag} / \mathrm{AgCl}$ as a reference electrode. The cyclic voltammetry was carried out in freshly distilled dichloromethane (DCM) using $0.1 \mathrm{M}$ tetrabutylammonium hexaflurophosphate $\left(\mathrm{TBAPF}_{6}\right)$ as supporting electrolyte and a scan rate of $50 \mathrm{mV} \mathrm{sec}^{-1}$ was used. The cyclic voltammogram was calibrated with $\mathrm{Fc} / \mathrm{Fc}^{+}$redox couple. The HOMO energy level of newly synthesized molecule PY-II was calculated by photoelectron spectroscopy in air (PESA) using a spin coated thin film of PY-II on the ITO coated glass substrate. The surface of PY-II thin film was irradiated with UV energy that emits photoelectrons of certain energy level known as photoelectron work function. The photoelectron output is plotted with horizontal $\mathrm{X}$-axis as the UV energy applied and the vertical $\mathrm{Y}$-axis as the standardized photoelectron yield ratio, the result is a line with a specific slope of degree $(\mathrm{Y} / \mathrm{eV})$. The HOMO value of PY-II was calculated from the onset energy level recorded in PESA. Solution and thin film (solid state) photoluminescence quantum yield (PLQY) measurements were carried out with an Absolute PL Quantum Yield Spectrometer (Hamamatsu, C11347) using an integrating sphere equipped with a multichannel spectrometer. ${ }^{48}$ The roughness measurement was conducted by atomic force microscopy (AFM). PY-II solution prepared in chloroform was spin-coated on an ITO substrate. It was baked at $70{ }^{\circ} \mathrm{C}$ for $20 \mathrm{~min}$. The scan dimension was typically set as 5 x $5 \mu \mathrm{m}$.

\section{Synthesis of 9-(5-bromopentyl)-9H-carbazole (2)}

Into a $100 \mathrm{~mL}$ round bottom flask equipped with a magnetic stirring bar and a dropping funnel were placed carbazole 1 ( $2 \mathrm{~g}$, $11.96 \mathrm{mmol}), \mathrm{KOH}(0.671 \mathrm{~g}, 11.96 \mathrm{mmol})$ and DMF (40 mL). The reaction mixture was stirred at room temperature for $1 \mathrm{~h}$. 1,5-Dibromopentane $(8.25 \mathrm{~g}, 35.88 \mathrm{mmol})$ was added dropwise into the reaction mixture at $0{ }^{\circ} \mathrm{C}$. After the addition, the reaction mixture was allowed to stir at room temperature for $12 \mathrm{~h}$. The reaction mixture was diluted with water $(50 \mathrm{~mL})$ and extracted with dichloromethane $(100 \mathrm{~mL})$. The organic layer was washed with water, brine solution, and again with water. The organic layer was dried over anhydrous sodium sulfate and filtered. Removal of the solvent on a rotary evaporator yielded a crude product which was purified by column chromatography using petroleum ether: dichloromethane $(9: 1, \mathrm{v} / \mathrm{v})$ as an eluent to give compound 2 as a white powder. (2.95 g, yield $78 \%)$. ${ }^{1} \mathrm{H}$ NMR $\left(400 \mathrm{MHz}, \mathrm{CDCl}_{3}\right) \delta=8.12(\mathrm{~d}, J=7.3 \mathrm{~Hz}, 2 \mathrm{H}), 7.49$ (t, $J=$ $7.6 \mathrm{~Hz}, 2 \mathrm{H}), 7.41(\mathrm{~d}, J=7.9 \mathrm{~Hz}, 2 \mathrm{H}), 7.31-7.19(\mathrm{~m}, 2 \mathrm{H})$, 4.34 (t, $J=7.0 \mathrm{~Hz}, 2 \mathrm{H}), 3.37$ (t, $J=6.7 \mathrm{~Hz}, 2 \mathrm{H}), 2.00-1.82$ $(\mathrm{m}, 4 \mathrm{H}), 1.62-1.46(\mathrm{~m}, 2 \mathrm{H}) .{ }^{13} \mathrm{C} \mathrm{NMR}\left(100 \mathrm{MHz}, \mathrm{CDCl}_{3}\right) \delta=$ $140.27,125.61,122.80,120.34,118.80,108.51,42.72,33.28$, $32.40,28.11,25.83$. 
Synthesis of 9-(5-(4-iodophenoxy)pentyl)-9H-carbazole (3) Into a $100 \mathrm{~mL}$ round bottom flask equipped with a magnetic stirring bar and a dropping funnel were placed 4-iodophenol (2 g, $9.09 \mathrm{mmol}), \mathrm{KOH}(36.36 \mathrm{mmol})$ and DMSO (30 mL). After stirring for 10 minutes, the solution of 9-(5-bromopentyl)-9Hcarbazole (3.16 g, $10 \mathrm{mmol})(2)$ in DMSO $(20 \mathrm{~mL})$ was added dropwise to the reaction mixture. The reaction mixture was stirred at room temperature for $24 \mathrm{~h}$. The reaction mixture was poured into water $(50 \mathrm{~mL})$ and extracted with dichloromethane $(100 \mathrm{~mL})$. The organic layer was separated and washed with water, brine solution and again with water. The organic layer was dried over anhydrous sodium sulfate and filtered. Removal of solvent on a rotary evaporator yielded a crude product which was purified by column chromatography using petroleum ether: dichloromethane $(8: 2, v / v)$ as an eluent to give compound $\mathbf{3}$ as colorless crystals. (3.72 g, yield $82 \%) .{ }^{1} \mathrm{H}$ NMR $(400 \mathrm{MHz}$, $\left.\mathrm{CDCl}_{3}\right) \delta=8.14(\mathrm{~d}, \mathrm{~J}=7.78 \mathrm{~Hz}, 2 \mathrm{H}), 7.58-7.45(\mathrm{~m}, 4 \mathrm{H}), 7.44-$ $7.36(\mathrm{~m}, 2 \mathrm{H}), 7.27$ (t, $J=7.33 \mathrm{~Hz}, 2 \mathrm{H}), 6.67-6.55(\mathrm{~m}, 2 \mathrm{H}), 4.33$ $(\mathrm{t}, \mathrm{J}=7.10 \mathrm{~Hz}, 2 \mathrm{H}), 3.84$ (t, J=6.18 Hz, 2H), 2.02-1.88 (m, 2H), $1.84-1.72(\mathrm{~m}, 2 \mathrm{H}), 1.59-1.47(\mathrm{~m}, 2 \mathrm{H}) .{ }^{13} \mathrm{C}$ NMR $(100 \mathrm{MHz}$, $\left.\mathrm{CDCl}_{3}\right) \delta=158.75,140.30,138.09,125.59,122.77,120.33$, $118.76,116.83,108.55,82.53,67.59,42.81,28.89,28.71$, 23.77.

Synthesis of 9-(5-(4-(4,4,5,5-tetramethyl-1,3,2-dioxaborolan2-yl)phenoxy)pentyl)-9H-carbazole (4)

Into a $50 \mathrm{~mL}$ Schlenk tube were added 9-(5-(4Iodophenoxy)pentyl)-9H-carbazole (3) (1 g, $2.20 \mathrm{mmol})$, bis(pinacolato)diboron ( $0.669 \mathrm{~g}, 2.60 \mathrm{mmol}), \mathrm{PdCl}_{2}$ (dppf) (161 $\mathrm{mg}, 0.2 \mathrm{mmol})$ and potassium acetate $(646 \mathrm{mg}, 6.60 \mathrm{mmol})$ under argon flow and then the tube was evacuated for half an hour. Then, under an argon flow, anhydrous dioxane $(10 \mathrm{~mL})$ was added to the reaction mixture. The reaction mixture was stirred at room temperature for $1 \mathrm{~h}$ and then at $80{ }^{\circ} \mathrm{C}$ for $24 \mathrm{~h}$. The reaction was quenched by addition of ice-cold water $(100$ $\mathrm{mL})$ and extracted with dichloromethane $(200 \mathrm{~mL})$. The combined organic layers were washed with water, brine solution and again with water. The organic layer was dried over anhydrous sodium sulfate and filtered. Removal of the solvent on a rotary evaporator yielded a crude product, which was chromatographed on neutral alumina using $2 \%$ ethyl acetate in petroleum ether as an eluent to give compound $\mathbf{4}$ as a white solid (640 mg, yield 64\%). ${ }^{1} \mathrm{H}$ NMR (400 MHz, $\left.\mathrm{CDCl}_{3}\right) \delta=$ $8.11(\mathrm{~d}, J=7.71 \mathrm{~Hz}, 2 \mathrm{H}), 7.75(\mathrm{~d}, J=8.46 \mathrm{~Hz}, 2 \mathrm{H}), 7.52-7.35$ (m, 4H), 7.29-7.18 (m, 2H), $6.85(\mathrm{~d}, J=8.59 \mathrm{~Hz}, 2 \mathrm{H}), 4.31$ (t, $\mathrm{J}=6.95 \mathrm{~Hz}, 2 \mathrm{H}), 3.91(\mathrm{t}, \mathrm{J}=6.32 \mathrm{~Hz}, 2 \mathrm{H}), 2.04-1.87(\mathrm{~m}, 2 \mathrm{H})$, $1.85-1.70(\mathrm{~m}, 2 \mathrm{H}), 1.68-1.50(\mathrm{~m}, 2 \mathrm{H}), 1.34(\mathrm{~S}, 12 \mathrm{H}) .{ }^{13} \mathrm{C} \mathrm{NMR}$ $\left(100 \mathrm{MHz}, \mathrm{CDCl}_{3}\right) \delta=161.52,140.33,136.47,125.59,122.78$, $120.32,118.75,113.80,108.57,83.49,67.30,42.85,28.97$, $28.73,24.82,23.82$.

Synthesis of 1,3,6,8-tetrakis(4-((5-(9H-carbazol-9yl)pentyl)oxy)phenyl)pyrene (PY-II)

Into a $50 \mathrm{~mL} \quad$ Schlenk tube were placed 1,3,6,8tetrabromopyrene $\quad(0.1 \mathrm{~g}, \quad 0.19 \mathrm{mmol}), \quad 9-(5-(4-(4,4,5,5-$ tetramethyl-1,3,2-dioxaborolan-2-yl)phenoxy)pentyl)-9H-

carbazole $(0.528 \mathrm{~g}, 1.16 \mathrm{mmol}), 2 \mathrm{M} \mathrm{K}_{2} \mathrm{CO}_{3}(2 \mathrm{ml})$ and $\mathrm{Pd}\left(\mathrm{PPh}_{3}\right)_{4}(22 \mathrm{mg}, 0.019 \mathrm{mmol})$ under argon flow. The tube was evacuated and refilled with argon three times and then toluene $(10 \mathrm{~mL})$ was added into the flask. The reaction mixture was stirred at $80{ }^{\circ} \mathrm{C}$ for $48 \mathrm{~h}$ and then extracted with dichloromethane $(3 \times 50 \mathrm{~mL})$. The combined organic layer was washed with water, brine solution and again with water. The organic layer was dried over sodium sulfate and filtered. Removal of the solvent on a rotary evaporator yielded a crude product, which was purified by column chromatography using petroleum ether: dichloromethane $(6: 4, \mathrm{v} / \mathrm{v})$ as an eluent to give title compound PY-II as a yellow solid (181 mg, yield 62\%). ${ }^{1} \mathrm{H} \mathrm{NMR}\left(400 \mathrm{MHz}, \mathrm{CDCl}_{3}\right) \delta=8.22-8.04(\mathrm{~m}, 12 \mathrm{H}), 7.95(\mathrm{~s}$, $2 \mathrm{H}), 7.58$ (d, $J=7.9 \mathrm{~Hz}, 8 \mathrm{H}), 7.53-7.42$ (m, $16 \mathrm{H}), 7.24$ (d, $J$ $=7.3 \mathrm{~Hz}, 8 \mathrm{H}), 7.04(\mathrm{~d}, J=7.9 \mathrm{~Hz}, 8 \mathrm{H}), 4.39(\mathrm{t}, J=6.7 \mathrm{~Hz}, 8$ $\mathrm{H}), 4.04(\mathrm{t}, J=5.8 \mathrm{~Hz}, 8 \mathrm{H}), 2.10-1.97(\mathrm{~m}, 8 \mathrm{H}), 1.96-1.84$ $(\mathrm{m}, 8 \mathrm{H}), 1.71-1.60(\mathrm{~m}, 8 \mathrm{H}){ }^{13} \mathrm{C}$ NMR $\left(100 \mathrm{MHz}, \mathrm{CDCl}_{3}\right) \delta=$ $158.4,140.4,136.7,133.5,131.7,129.6,128.0,126.1,125.6$, 125.1, 122.8, 120.4, 118.8, 114.4, 108.6, 67.7, 42.9, 29.1, 28.8, 23.9. MALDI-TOF: Calculated 1510.7275 , found 1511.4016 .

\section{OLED Device Fabrication and Characterization}

OLED devices were fabricated using PY-II active emitting layer for evaluating the electroluminescent (EL) performance. The OLED device configuration glass/ITO/PEDOT: PSS/PYII/TPBi/LiF/Al was used. Patterned ITO glass substrates were routinely cleaned, blown dry by nitrogen gas and then treated with ultra-violet ozone. The filtered poly (3, 4ethylenedioxythiophene) polystyrene sulfonate (PEDOT: PSS) solution was spin-coated on the patterned ITO glass substrates and baked at $140{ }^{\circ} \mathrm{C}$ for $20 \mathrm{~min}$. Then PY-II solution prepared in chloroform was spin-coated on PEDOT: PSS coated ITO glass substrates and baked at $70{ }^{\circ} \mathrm{C}$ for $20 \mathrm{~min}$. All the spin-coat processes were performed in nitrogen glove box environment. PY-II coated substrates were then loaded into a thermal evaporation chamber at a vacuum of $2 \times 10^{-6}$ Torr. The layers of $20 \mathrm{~nm}$ of 2, 2', 2"-(1, 3, 5-benzinetriyl)-tris (1-phenyl-1-Hbenzimidazole) (TPBi), $1 \mathrm{~nm}$ of lithium fluoride (LiF) and 200 $\mathrm{nm}$ of aluminium (Al) were coated on the substrates in that sequence. The voltage-current-brightness (I-V-B) characteristics, the C.I.E. coordinates, the EL emission spectra were measured by a Spectra PR650 CCD camera with a computer controlled power supply. The current and power efficiencies were calculated from I-V-B data of the devices.

\section{Results and Discussion}

Initially, commercially available carbazole was converted to 9(5-bromopentyl)-9H-carbazole (2) via alkylation reaction with excess 1,5-dibromopentane. The compound 2 was subsequently reacted with 4-iodophenol to afford 9-(5-(4iodophenoxy)pentyl)-9H-carbazole (3). The transformation of compound 3 to the boronic ester analog 9-(5-(4-(4,4,5,5tetramethyl- 1,3,2-dioxaborolan-2-yl)phenoxy)pentyl)-9Hcarbazole (4) was carried out via Miyaura borylation reaction. The target compound 1,3,6,8-tetrakis(4-((5-(9H-carbazol-9yl)pentyl)oxy)phenyl)pyrene (PY-II) was prepared via the classical Suzuki coupling using 1,3,6,8-tetrabromopyrene and 
monoboronic ester analogue 4. The synthesis of PY-II is depicted in Scheme 1. PY-II was readily soluble in common organic solvents such as dichloromethane, chloroform, toluene and tetrahydrofuran at room temperature. The chemical structure of PY-II and intermediate derivatives were confirmed by ${ }^{1} \mathrm{H}$ NMR, ${ }^{13} \mathrm{C}$ NMR and MALDI-TOF spectroscopy (see $\mathrm{ESI} \dagger$ for further details). The thermal properties of PY-II were studied by thermogravimetric analysis (TGA) and differential scanning colorimetry (DSC). TGA and DSC curves are shown in ESI. $\dagger$

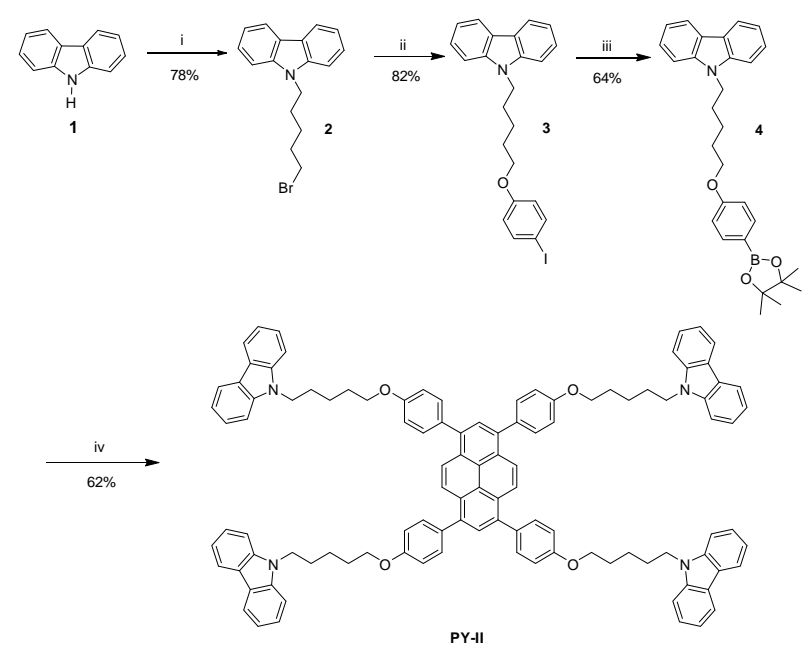

Scheme 1 Synthesis of PY-II. Reagents and conditions: i) 1,5dibromopentane, $\mathrm{KOH}, \mathrm{DMF}, \mathrm{rt}, 12 \mathrm{~h}$; ii) 4-iodophenol, $\mathrm{KOH}$, DMSO, rt, 24 h; iii) bis(pinacolato)diboron, $\mathrm{Pdcl}_{2}$ (dppf), AcOK, 1,4dioxane, $80{ }^{\circ} \mathrm{C}, 24 \mathrm{~h}$; iv) 1,3,6,8-tetrabromopyrene, $\mathrm{Pd}\left(\mathrm{PPh}_{3}\right)_{4}$, $\mathrm{K}_{2} \mathrm{CO}_{3}$, aliquat, toluene, $80{ }^{\circ} \mathrm{C}, 48 \mathrm{~h}$.

PY-II showed high thermal stability with initial decomposition at $345{ }^{\circ} \mathrm{C}$ and $5 \%$ weight loss at $435^{\circ} \mathrm{C}$. In DSC analysis PYII exhibited a sharp melting peak at $215^{\circ} \mathrm{C}$ during the first heating scan. However, after cooling and reheating of sample during the second heating scan, it demonstrated the molecular glass behaviour with glass transition temperature $\left(\mathrm{T}_{\mathrm{g}}\right)$ at $82{ }^{\circ} \mathrm{C}$. Further heating above $\mathrm{T}_{\mathrm{g}}$ did not result in any other thermal transitions indicating the formation of stable amorphous glass.

Density Functional Theory (DFT) ${ }^{49}$ calculations were performed to compute molecular structures, the energies of the frontier orbitals and their localization as well as absorption and photoluminescence spectra. The calculations were carried out using $\mathrm{B}^{2} \mathrm{LYP}^{50}$ (for frontier orbital energies and optical properties) and CAM-B3LYP ${ }^{51}$ (for the oxidation potential) with the LANL2DZ basic set. The absorption and PL spectra were computed with time dependent DFT (TD-DFT) considering the lowest six excited states. ${ }^{52}$ The PL spectrum was computed by optimizing the first excited state. The calculations were performed with the polarizable continuum model (PCM) of the chloroform solvent. ${ }^{53}$ The calculations were performed using Gaussian 09. ${ }^{54}$ The HOMO (highest occupied molecular orbital) and LUMO (lowest unoccupied molecular) of PY-II are shown in Fig. 2.

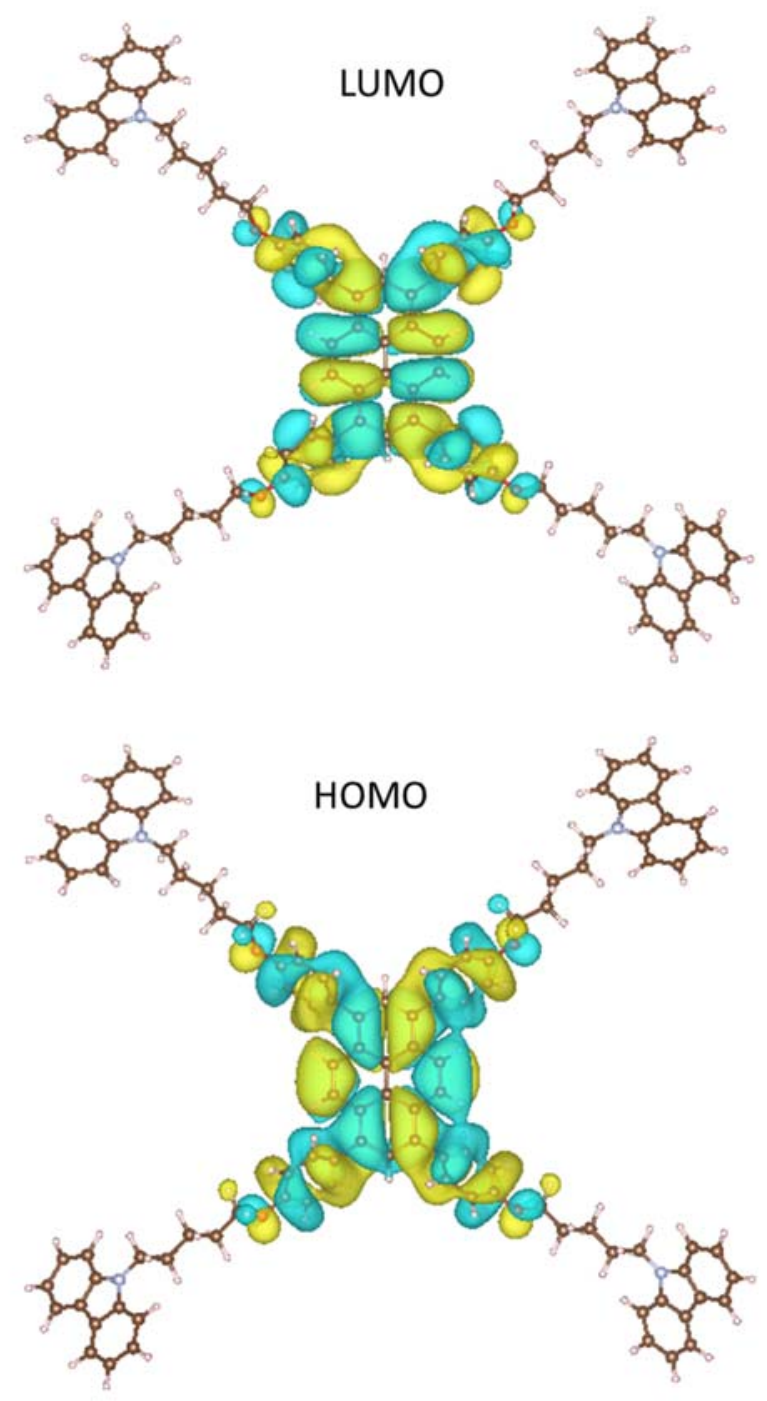

Fig. 2 Distributions of HOMO and LUMO of PY-II obtained with DFT.

The HOMO and LUMO are centered on the pyrene core. The HOMO is only slightly more delocalized than LUMO and has appreciable amplitude on the oxyphenylene moieties, while the LUMO is localized on the central pyrene core and partially on the oxyphenylene moieties. The theoretically predicted HOMO and LUMO values are $-5.18 \mathrm{eV}$ and $-1.96 \mathrm{eV}$, respectively. The HOMO-LUMO bandgap for PY-II was calculated to be 3.22 eV. The absorption and PL peak maximum of PY-II computed by B3LYP are $424 \mathrm{~nm}$ and $523 \mathrm{~nm}$, respectively. The absorption and PL spectra are shown in ESI. $\dagger$ It was previously shown that a functional with a significant fraction of exact exchange is required to accurately estimate the oxidation potential. ${ }^{55,56}$ The oxidation potential was therefore estimated using the range-separated hybrid functional CAM-B3LYP from the difference in energies of (respectively optimized) neutral and cationic states, ${ }^{55,56}$ giving the value of $5.44 \mathrm{eV}$, which is in good agreement with the experimental value (see below). 
The optical properties of PY-II were characterized by UVvis absorption and photoluminescence spectroscopy. The absorption spectra of PY-II in chloroform solution and in solid state are shown in Fig. 3 (upper). The absorption in the range $275 \mathrm{~nm}$ to $500 \mathrm{~nm}$ clearly indicated wide band gap nature of PY-II. In solution, PY-II exhibited absorption peaks at $297 \mathrm{~nm}$ and $394 \mathrm{~nm}$ which are originated from $\pi-\pi^{*}$ transition of carbazole moieties and pyrene core, respectively.
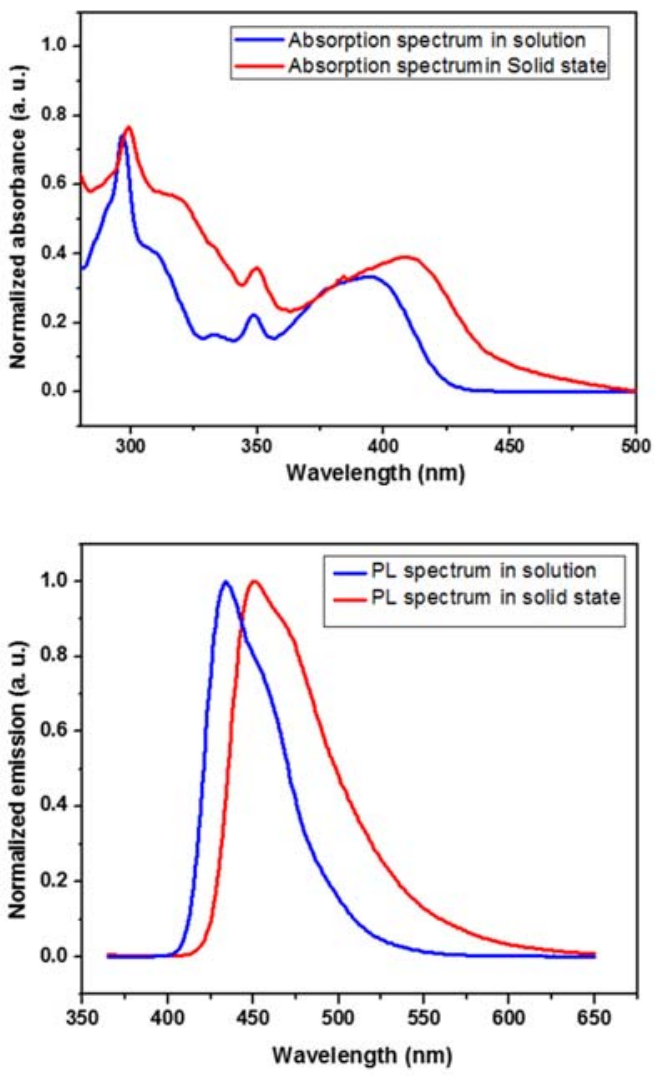

Fig. 3 Absorption (top) and Photoluminescence (bottom) spectra of PY-II in solution and solid state

When compared with the absorbance peak of molecular pyrene $(338 \mathrm{~nm})$, the absorption peak at $394 \mathrm{~nm}$ exhibited red shift of $56 \mathrm{~nm}$, which could be attributed to extended conjugation length between pyrene core and the attached oxyphenylene moiety. The absorption spectrum in solid state was nearly identical to that in solution with absorption peaks at $298 \mathrm{~nm}$ and $408 \mathrm{~nm}$. The optical band gap calculated from absorption onset value from the thin film spectrum was found to be 2.76 $\mathrm{eV}$. This higher band gap value of PY-II is a clear indication of a blue emitter. The photoluminescence (PL) spectra of PY-II in both solution and solid state are shown in Fig. 3 (bottom panel). The emission peaks at $434 \mathrm{~nm}$ and $450 \mathrm{~nm}$ were observed in solution and thin film of PY-II, respectively. The thin film emission at $450 \mathrm{~nm}$ is the most suitable blue emission colour for human eye. The shape of the emission spectrum in thin film is almost similar to that in solution with slight red shift of 16 $\mathrm{nm}$. This result clearly indicated little intermolecular interaction between the emissive cores which could be attributed to the encapsulation effect of the alkyl separated carbazole moiety.
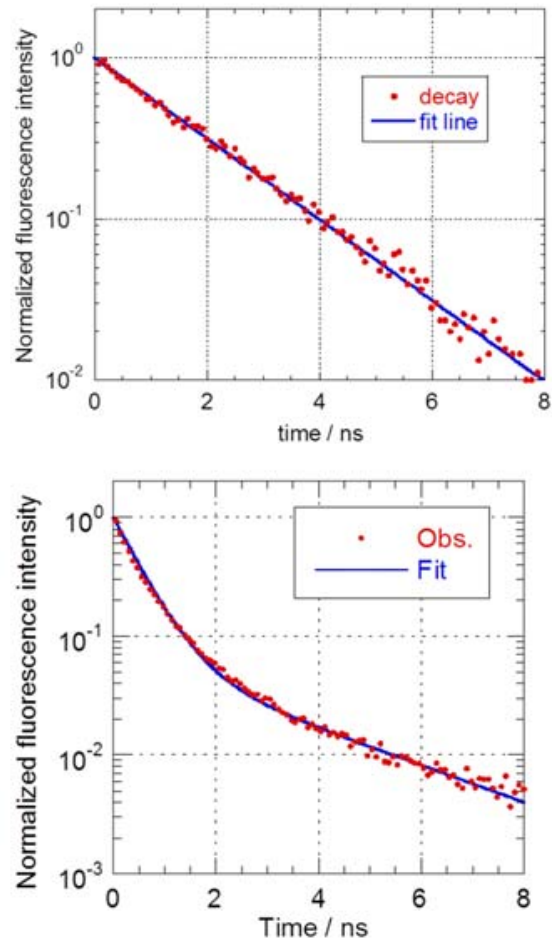

Fig. 4 Transient PL decay curves of PY-II in solution (a) and thin film (b)

The PL quantum yield (PLQY) of PY-II in chloroform solution and thin film were measured by using integrating sphere technique after argon gas bubbling for the solution sample and under ambient atmosphere for film sample. In solution PY-II exhibited a very high PLQY of almost 0.9. In our samples, there are two fluorophores namely pyrene (Py) and carbazole (Ca). Absorption band of $\mathrm{Ca}$ is located higher than that of Py. If there is no energy transfer occurs between $\mathrm{Py}$ and $\mathrm{Ca}$ fluorophores then they emit fluorescence independently. In such scenario, CA moiety can be preferably excited by shorter wavelength light and Py moiety can be preferably excited by longer wavelength light. If PLQY of pyrene moiety is higher than that of carbazole moiety, PLQY at shorter wavelength excitation is smaller than PLQY at longer wavelength excitation because fraction of excited Py (or Ca) moiety is different. This is not the case in our present study that's why PLQY values were not sensitive to the excitation wavelength between $300 \mathrm{~nm}$ and $400 \mathrm{~nm}$. In this excitation wavelength range, absorption due to pyrene and carbazole moieties coexists. Since absorption due to pyrene moiety is located at longer wavelength range, fluorescence spectra shown in Fig. 3 is assigned to pyrene fluorescence. Accordingly, change of PLQY and spectral shape are expected to be observed, if carbazole moiety is excited preferably at shorter wavelength range. As mentioned above, no change of PLQY and spectral 
shape was observed in this excitation wavelength range. This clearly shows that energy transfer from the excited carbazole moiety to the pyrene part occurs efficiently and subsequently fluorescence emission occurs with high PLQY. In other words, all excitation energy obtained by light absorption is converted to the excitation energy of Py. Thus, PLQY value is determined by PLQY of pyrene moiety. We also observed spectral shape is not sensitive to excitation wavelength. This again indicates the presence of energy transfer from CA to Py because only Py gives fluorescence. However, the PLQY value in thin film was smaller than that in solution with the value of 0.33 , suggesting some deactivation processes are induced. For further insight into excited-state relaxation processes, time-resolved PL decay measurements were carried out in chloroform solution and thin film (Fig 4). In solution, PY-II exhibited a simple exponential decay with a fluorescence lifetime $(\tau)$ of $1.75 \mathrm{~ns}$, while in thin film double exponential decay profile was observed with a lifetime of $0.5 \mathrm{~ns}(93 \%)$ and $2.8 \mathrm{~ns}(7 \%)$ for fast and slow components, respectively. The fast component spectrum of film was similar to that in solution. This suggests that the fast component is originated from the pyrene moiety and quenched through some decay process, judging from the shorter lifetime. After decay of the fast component fluorescence, the slow component fluorescence was observed (Fig. S12) at longer wavelength range.

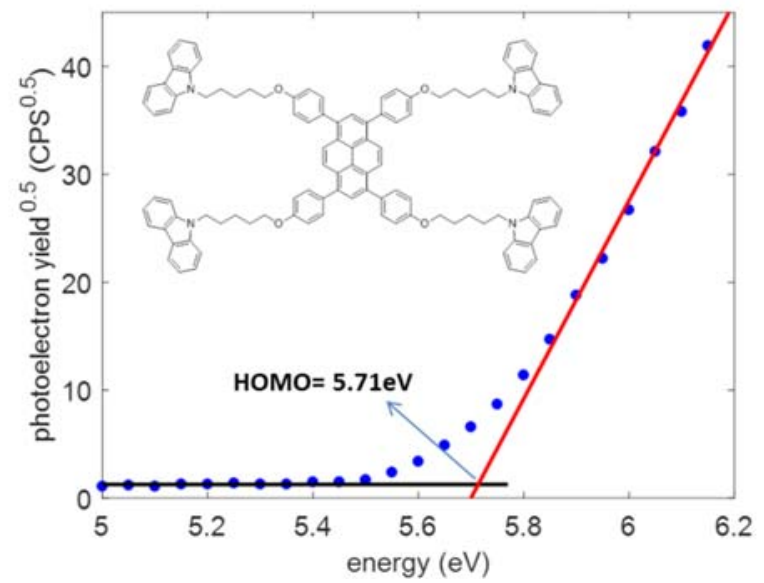

Fig. 5 Photoelectron spectroscopy in air (PESA) analysis of PY-II in thin film.

This is a possible reason for the slight difference of spectral shape between the solution and the film, which suggests that energy transfer takes place from the pyrene moiety to some defect site, presumably to the aggregates of carbazole moieties. The high photoluminescence in solution of PY-II is an attractive signature to use this fluorescent material with high brightness not only in photonic applications but also in other applications such as bioimaging or chemical sensing where high solution quantum yield is desired.

The HOMO level of PY-II was characterized by photoelectron spectroscopy in air (PESA) as shown in Fig. 5. Thin film of PY-II was spin coated on an ITO substrate, which was used for determination of ionization potential. The photoelectron yield ratio was measured with respect to applied UV-energy. The onset value calculated from this graph gave HOMO value of $-5.71 \mathrm{eV}$. Additionally, the HOMO level of PY-II was investigated using cyclic voltammetry and voltammogram is shown in ESI. $\uparrow$ The HOMO level calculated from onset oxidation potential $(-0.84 \mathrm{eV})$ was found to be -5.64 $\mathrm{eV}$, which is in good agreement with the HOMO level (-5.71 eV) calculated by PESA. Such a low HOMO value could be attributed to the presence of a non-conjugated aliphatic spacer in the structure, which is important for obtaining air stable organic electronic devices. The device configuration using compound PY-II as an active emissive layer is shown in Figure 6 with ITO/PEDOT:PSS(50nm)/PY-II (35 nm)/TPBi (20 $\mathrm{nm}) / \mathrm{LiF}(1 \mathrm{~nm}) / \mathrm{Al}(200 \mathrm{~nm})$ device architecture. ITO coated glass and $\mathrm{LiF} / \mathrm{Al}$ act as anode and cathode, respectively, whereas TPBi is an electron transporting layer.

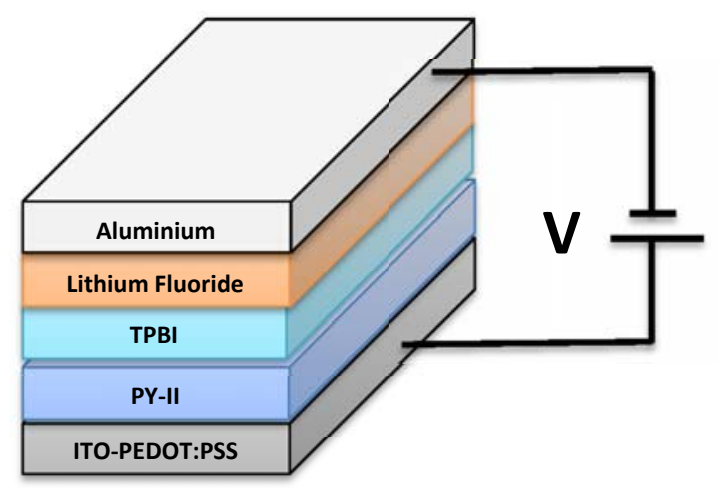

Fig. 6 The OLED device architecture using PY-II.

Table 1. EL performance summary of PY-II based OLED devices.

\begin{tabular}{|c|c|c|c|c|c|}
\hline $\begin{array}{c}\text { Turn on } \\
\text { voltage } \\
\text { (V) } \\
@ 1 \mathbf{c d} / \mathbf{m}^{2}\end{array}$ & $\begin{array}{c}\text { Current } \\
\text { efficiency } \\
\text { (Cd/A) }\end{array}$ & $\begin{array}{c}\text { Power } \\
\text { efficiency } \\
\text { (Im/W) }\end{array}$ & CIE & $\begin{array}{c}\text { EL } \\
\text { peak } \\
(\mathbf{n m})\end{array}$ & $\begin{array}{c}\text { Max. } \\
\text { Brightness } \\
\left(\mathbf{c d} / \mathbf{m}^{2}\right)\end{array}$ \\
\hline 4.5 & 0.41 & 0.17 & $\begin{array}{c}(0.16, \\
0.16)\end{array}$ & $\begin{array}{c}445, \\
470\end{array}$ & 202 \\
\hline
\end{tabular}

When a potential is applied, holes are injected from the anode and electrons are injected from the cathode, respectively. Due to recombination of holes and electrons in the active emissive layer, blue photon emission was observed in the OLED devices. The obtained OLED device performance parameters such as turn on voltage, current efficiency, power efficiency, CIE coordinates, electroluminescent peak and maximum brightness and their values are given in Table 1. The observed electroluminescence (EL) spectrum is shown in Fig. 7 (bottom panel). PY-II device exhibited an EL maxima of $445 \mathrm{~nm}$ and $470 \mathrm{~nm}$ emission peaks in visible blue region with a full wave half maximum (FWHM) of $70 \mathrm{~nm}$ and CIE coordinates of 0.16 and 0.16. We have noticed that PY-II exhibits different EL 
spectrum than PL spectrum. We measured the EL spectra of the PY-II device from 5-11V, all spectra contain two emission peaks, one at 450 and other at $470 \mathrm{~nm}$. The difference between the PL and EL spectra is attributed to the voltage effect and this can be clearly seen in the applied voltage-EL graph (See S13). PY-II device showed a turn on voltage $\left(\mathrm{V}_{\text {on }}\right)$, maximum current efficiency ( $\left.\mathrm{CE}_{\max }\right)$, maximum power efficiency ( $\mathrm{PE}_{\max }$ ), maximum brightness $\left(\mathrm{B}_{\max }\right)$ of $4.5 \mathrm{~V}, 0.41 \mathrm{~cd} / \mathrm{A}, 0.17 \mathrm{~lm} / \mathrm{W}$ and $202 \mathrm{~cd} / \mathrm{m}^{2}$, respectively. The details of $\mathrm{j}-\mathrm{V}-\mathrm{B}, \mathrm{CE}$ and $\mathrm{PE}$ characteristics of PY-II device are shown in Fig. 7 (top panel) and ESI, $\uparrow$ respectively. As the voltage was increased from 4.5 $\mathrm{V}$ to $7 \mathrm{~V}$ and $8.5 \mathrm{~V}$, the CE and PE of PY-II device increased rapidly to $C E_{\max }$ and $P E_{\max }$ of $0.41 \mathrm{~cd} / \mathrm{A}$ and $0.17 \mathrm{~lm} / \mathrm{W}$, respectively. As the drive voltage was further increased, $\mathrm{CE}$ and PE curves of PY-II device typically roll-off. However, CE and PE of PY-II device still remained at higher than one-half of the $\mathrm{CE}_{\max }$ and $\mathrm{PE}_{\max }$ even as the drive voltage was further increased beyond $10 \mathrm{~V}$. The stable blue colour output additionally proves that PY-II has high prospective to be a blue emitter in OLED applications. The turn on voltage of $4.5 \mathrm{~V}$ also makes PY-II based OLED device energy efficient. The detailed summary of OLED device is shown in Table 1.

The morphological behaviour of the PY-II active layer thin film was characterized by AFM as shown in ESI. $\dagger$ The measured root mean square (RMS) roughness of film samples is $3.46 \mathrm{~nm}$. The pristine ITO coated glass showed surface roughness with an RMS of $3.25 \mathrm{~nm}$. PY-II exhibited a low roughness value, which is desirable for transportation of holes and electrons in the recombination zone.
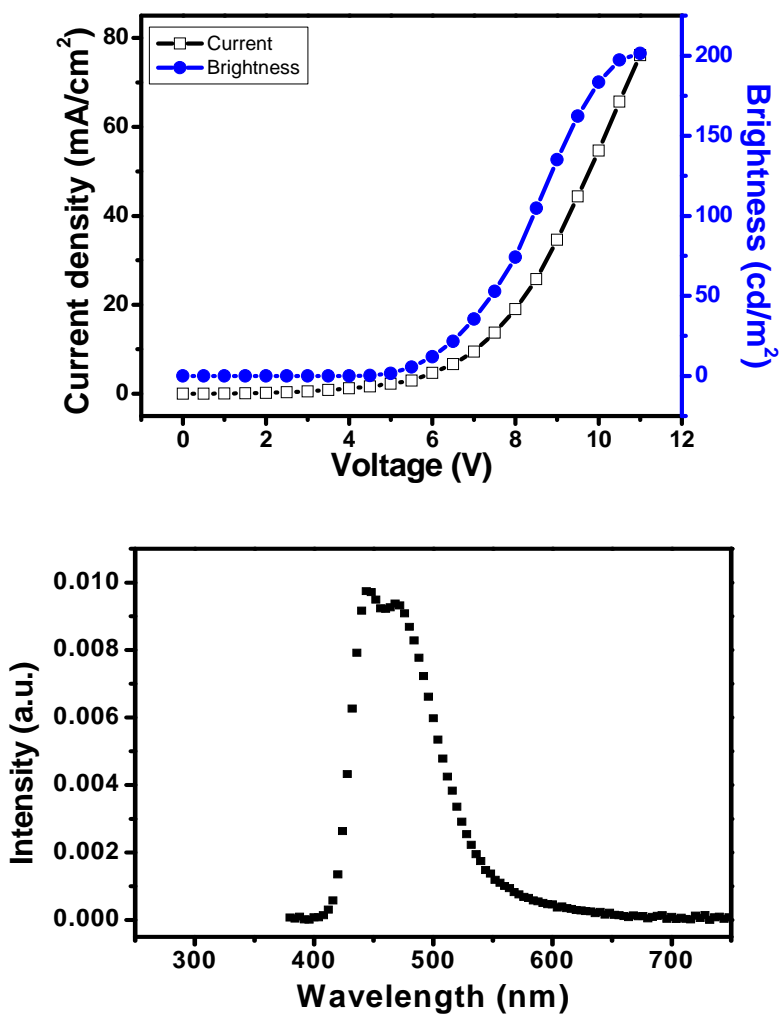

Fig. 7 Current- voltage-brightness characteristics (top) and electroluminescence spectrum of PY-II (bottom)

\section{Conclusions}

In summary, we demonstrated the design strategy and synthesis of a new pyrene cored small organic molecule, namely, PY-II containing carbazole moiety with alkyl spacer for solution processable OLEDs. PY-II exhibited excellent solution processability and high thermal stability. PY-II showed wide absorption in the range $275 \mathrm{~nm}$ to $500 \mathrm{~nm}$ with an optical band gap of $2.76 \mathrm{eV}$. The PLQY of PY-II was found to be 0.90 and 0.33 in solution and solid state, respectively. The HOMO level calculated using PESA was found to be $-5.71 \mathrm{eV}$. The solution processed non-doped OLED device using PY-II as EML exhibited turn on voltage of $4.5 \mathrm{~V}$, blue emission with EL peaks at $445 \mathrm{~nm}$ and $470 \mathrm{~nm}$, a maximum brightness of $202 \mathrm{~cd} / \mathrm{m}^{2}$ and a power efficiency of $0.17 \mathrm{~lm} / \mathrm{W}$. These results support that this class of functional materials hold a high potential for future cost effective and efficient light emitting materials for OLED devices. We believe that further improvements with introduction of a larger number of conjugated moieties on the pyrene core would greatly improve the performance of molecular and device structure. The higher PLQY and higher brightness in solution are important attributes for bioimging and sensor applications where PY-II in solution can be used. Overall, the present study pinpoints a useful strategy to design new solution processable pyrene-based materials as blue emitters with bright photoluminescence.

\section{Acknowledgement}

F. L. Wong and V. A. L. Roy are thankful to Research Grants Council of Hong Kong Special Administrative Region (Project No. T23-713/11). P.S. is thankful to QUT for the financial support from the Australian Research Council (ARC) for the Future Fellowship (FT130101337) and QUT core funding (QUT/ 322120-0301/07). S. M. is supported by the Ministry of Education of Singapore (AcRF Tier 1 grant). KF thanks the Australian Renewable Energy Agency for financial support.

\section{References}

1. C.W. Tang and S. A. Vanslyke, Appl. Phys. Lett., 1987, 51, 913.

2. C. W. Tang, S. A. Vanslyke and C. H. Chen, J. Appl. Phys., 1989, 65, 3610.

3. J. H. Burroughes, D. D. C. Bradley, A. R. Brown, R. N. Marks, K. Mackay, R. H. Friend, P. L. Burns and A. B. Holmes, Nature, 1990, 347, 539.

4. L. S. Hung and C. H. Chen, Mater. Sci. Eng., R, 2002, 39, 143.

5. R.-P. Xu, Y.-Q. Li and J.-X. Tang. J. Mater. Chem. C, 2016, 4, 9116.

6. S. Feng, L. Duan, L. Hou, J. Qiao, D. Zhang, G. Dong, L. Wang, and Y. Qiu. J. Phys. Chem. C, 2011, 115, 14278. 
7. M. Zhang, S. Xue, W. Dong, Q. Wang, T. Fei, C. Gu and Y. Ma. Chem. Commun., 2010, 46, 3923.

8. M. Cai, T. Xiao, E. Hellerich, Y. Chen, R. Shinar and J. Shinar. Adv. Mater., 2011, 23, 3590.

9. Y. R. Cho, H. S. Kim, Y.-J. Yu and M. C. Suh. Scientific Reports, 2015, 5, 15903.

10. H. Wu, G. Zhou, J. Zou, C.-L. Ho, W.-Y. Wong, W. Yang, J. Peng, and Y. Cao. Adv. Mater., 2009, 21, 4181.

11. L. Duan, L. Hou, T.-W. Lee, J. Qiao, D. Zhang, G. Dong, L. Wang and Y. Qiu. J. Mater. Chem., 2010, 20, 6392-6407.

12. J.-H. Jou, S. Kumar, A. Agrawal, T.-H. Li and S. Sahoo. J. Mater. Chem. C, 2015, 3, 2974.

13. A. C. Grimsdale, K. L. Chan, R. E. Martin, P. G. Jokisz, and A. B. Holmes. Chem. Rev., 2009, 109, 897-1091.

14. X.-H. Zhu, J. Peng, Y. Cao and J. Roncali. Chem. Soc. Rev., 2011, 40, 3509.

15. E. Kozma, W. Mróz, F. Villafiorita-Monteleone, F. Galeotti, A. Andicsová-Eckstein, M. Catellani and C. Botta, RSC Adv., 2016, 6, 61175.

16. R. K. Konidena, K. R. J. Thomas, S. Sahoo, D. K. Dubey and J.-H. Joub, J. Mater. Chem. C, 2017, 5, 709.

17. K. S. Yook and J. Y. Lee, Adv. Mater., 26, 2014, 4218.

18. Y.-L. Deng, L.-S. Cui, Y. Liu, Z.-K. Wang, Z.-Q. Jiang and L.-S. Liao, J. Mater. Chem. C, 2016, 4, 1250.

19. J. N. Moorthy, P. Natarajin, P. Venkatakrishnan, D. F. Huang and T. J. Chow, Org. Lett., 2007, 9, 5215.

20. M. Y. Lo, C. G. Zhen, M. Lauters, G. E. Jabbour and A. Sellinger, J. Am. Chem. Soc., 2007, 129, 5808.

21. R. D. Xia, W. Y. Lai, P. A Levermore, W. Huang and D. D. C. Bradley, Adv. Funct. Mater., 2009, 19, 2844.

22. Z. J. Zhao, J. H. Li, X. P. Chen, X. M. Wang, P. Lu and Y. Yang, J. Org. Chem., 2009, 74, 383.

23. J. B. Birks, Photophysics of Aromatic Molecules, WileyInterscience, London, 1970.

24. J. R. Lackowicz, Principles of Fluorescence Spectroscopy, Kluwer Academic/Plenum Publishers, New York, 2nd edn, 1999, 595.

25. M. T. Figueira-Duarte and K. Mullen, Chem. Rev., 2011, 111,7260 .

26. J. K. Salunke, P. Sonar, F. L. Wong, V. A. L. Roy, C. S. Lee and P. P. Wadgaonkar, Phys. Chem. Chem. Phys., 2014, 16, 23320.

27. J. K. Salunke, F. L. Wong, K. Feron, S. Manzhos, M. F. Lo, D. Shinde, A. Patil, C. S. Lee, V. A. L. Roy, P. Sonar and P. P. Wadgaonkar, J. Mater. Chem. C, 2016, 4, 1009.

28. P. Sonar, M. S. Soh, Y. H. Cheng, J. T. Henssler and A. Sellinger, Org. Lett., 2010, 12, 3292.

29. R. K. Konidena, K. R. J. Thomas, M. Singh and J.-H. Jou, J. Mater. Chem. C, 2016, 4, 4246.

30. T. H. El-Assaad, M. Auer, R. Castan eda, K. M. Hallal, F. M. Jradi, L. Mosca, R. S. Khnayzer, D. Patra, T. V. Timofeeva, J.L. Bre'das, E. J. W. List-Kratochvil, B. Wex and B. R. Kaafarani, J. Mater. Chem. C, 2016, 4, 3041.

31. R. Zhang, Y. Zhao, T. Zhang, L. Xu and Z. Ni, Dyes and Pigments, 2016, 130, 106.

32. D. G. Vanga, M. Santra, A. Keerthi and S. Valiyaveettil, Org. Biomol. Chem., 2014, 12, 7914.
33. C.-H. Chang, C.-L. Ho, Y.-S. Chang, I-C. Lien, C.-H. Lin, Y.-W. Yang, J.-L. Liao and Y. Chi, J. Mater. Chem. C, 2013, 1, 2639.

34. F. Zhao , L. Zhu , Y. Liu , Y. Wang, D. Ma, Organic Electronics, 2015, 27, 207.

35. M.-Y. Chang, C.-H, Wang, S.-C. Lin, and Y.-F. Chen, J. Appl. Phys., 2009, 105, 064318.

36. J. Liang, L. Ying, F. Huang and Y. Cao, J. Mater. Chem. C, 2016, 4, 10993.

37. L. Chen, Z. Ma, J. Ding, L. Wang, X. Jing and F. Wang, Chem. Commun., 2011, 47, 9519.

38. J.-X. Cai, T.-L. Ye, X.-F. Fan, C.-M. Han, H. Xu, L.-L. Wang, D.-G. Ma, Y. Lin and P.-F. Yan, J. Mater. Chem., 2011, 21, 15405.

39. H. Xu, D.-H. Yu, L.-L. Liu, P.-F.Yan, L.-W. Jia, G.-M. Li, and Z.-Y. Yue, J. Phys. Chem., B, 2010, 114, 141.

40. X. Ban, W. Jiang, T. Lu, X. Jing, Q. Tang, S. Huang, K. Sun, B. Huang, B. Lin, and Y. Sun, J. Mater. Chem. C, 2016, 4, 8810 .

41. D. Xia, B. Wang, B. Chen, S. Wang, B. Zhang, J. Ding, L. Wang, X. Jing, and F. Wang, Angew. Chem. Int. Ed., 2014, 53, 1048 .

42. T. Keawin, C. Sooksai, N. Prachumrak, T. Kaewpuang, D. Muenmart, S. Namuangruk, S. Jungsuttiwong, T. Sudyoadsuk and V. Promarak, RSC Adv., 2015, 5, 16422.

43. P. Moonsin, N. Prachumrak, S. Namuangruk, S. Jungsuttiwong, T. Keawin, T. Sudyoadsuk and V. Promarak, J. Mater. Chem. C, 2014, 2, 5540.

44. J. Li, D. Liu, Y. Li, C.-S. Lee, H.-L. Kwong, and S. Lee, Chem. Mater. 2005, 17, 1208.

45. D. Curiel, M. Mas-Montoya, C.-H. Chang, P.-Y. Chen, C.W. Tai and A. Tarraga, J. Mater. Chem. C, 2013, 1, 3421.

46. S. J. Kim, Y. J. Kim, Y. H. Son, J. A. Hur, H. A. Um, J. Shin, T. W. Lee, M. J. Cho, J. K. Kim, S. Joo, J. H. Yang, G. S. Chae, K. Choi, J. H. Kwon and D. H. Choi, Chem. Commun., 2013, 49, 6788.

47. X. Wang, S. Wang, Z. Ma, J. Ding, L. Wang, X. Jing and F. Wang, Adv. Funct. Mater., 2014, 24, 3413.

48. H. Ishida, S. Tobita, Y. Hasegawa, R. Katoh and K. Nozaki, Coord. Chem. Rev., 2010, 254, 2449.

49. W. Kohn and L. J. Sham, Phys. Rev., 1965, 40, A1133.

50. A. D. Becke, J. Phys. Chem., 1993, 5648.

51. T. Yanai, D. Tew and N. Handy, Chem. Phys. Lett., 2004, 393, 51.

52. M. A. L. Marques, Annu. Rev. Phys. Chem., 2004, 55, 427.

53. J. Tomasi, B. Mennucci and R. Cammi, Chem. Rev., 2006, 105, 2999.

54. M. J. Frisch, Gaussian 09, Gaussian Inc., Wallingford, 2009.

55. S. M. Alam, Y. Chen, S. Manzhos Chem. Phys. Lett., 2016, 659, 270.

56. Y. Chen, S. Manzhos, J. Power Sources, 2016, 336, 126. 
